\section{Laboratory Medicine}

\title{
Non-cultural detection of rectal and pharyngeal gonorrhoea by the Gen-Probe ${ }^{\circledR}$ PACE® 2 Assay
}

\author{
H Young, J Anderson, A Moyes, A McMillan
}

Objective: To assess the sensitivity and specificity of the Gen-Probe ${ }^{\circledR}$ PACE $® 2$ assay, which uses a chemiluminescent labelled single-stranded DNA probe to detect gonococcal ribosomal RNA (rRNA), for the non-cultural detection of rectal and pharyngeal gonorrhoea in homosexual men.

Subjects: 161 homosexual men attending the Department of Genitourinary Medicine, Edinburgh Royal Infirmary during the latter half of 1995 and the first quarter of 1996.

Methods: Duplicate rectal and pharyngeal swabs were collected for culture on modified New York City (MNYC) medium and detection of gonococcal nucleic acid by the Gen-Probe assay. Repeatedly reactive Gen-Probe specimens from culture negative patients were also tested by the Gen-Probe competition assay (PCA).

Results: Of the 161 patients, $23(14.3 \%)$ gave a positive culture at one or both sites (rectum 10, throat 8 , rectum and throat 5 ) compared with $28(16 \cdot 7 \%)$ who gave a positive Gen-Probe result at one or both sites (rectum 9, throat 11, rectum and throat 8). After resolution of discrepant results by PCA the sensitivity, specificity, positive predictive value (PPV) and negative predictive value (NPV) of Gen-Probe was $94 \cdot 1 \%, 100 \%, 100 \%$ and $99 \cdot 3 \%$ for rectal specimens while the corresponding values for pharyngeal specimens were $86 \cdot 4 \%, 100 \%, 100 \%$, and $97.9 \%$. The sensitivity and NPV of rectal culture were $88.2 \%$ and $98.6 \%$ while the corresponding values for pharyngeal culture were $59 \%$ and $93.9 \%$. Gen-Probe was significantly more sensitive than throat culture $(p<0.05)$ but not rectal culture $(p>0.2)$. The average Relative Light Units (RLU) value for the cut-off was 386 (range 351-450) while the average for a positive result was 20306 (range 403-110 104): this was, however, significantly higher $(p=0.019)$ in rectal specimens 31325 (range 1705-1 10 104) than in throat specimens 10447 (range 403-15 633).

Conclusions: Gen-Probe ${ }^{\circledR}$ PACE $® 2$ assay is a sensitive and specific method for the detection of rectal and pharyngeal gonorrhoea. As the Gen-Probe assay may detect nucleic acid from non viable gonococci the clinical significance of a probe positive culture negative specimen from a patient without culture evidence of gonorrhoea at another site is uncertain and requires further consideration. Nevertheless a positive result does indicate exposure to infection and could be important in ensuring appropriate partner notification action. If non-cultural methods are used to screen for gonococcal infection cultures should be obtained from patients with positive results in order that the antibiotic susceptibility and molecular epidemiology of the gonococcal population can be monitored.

(Genitourin Med 1997;73:59-62)

Keywords: Neisseria gonorrhoeae; DNA probe; pharynx; rectum

\section{Introduction}

The epidemiology of gonorrhoea has changed in recent years with a much greater proportion of infections now acquired by homosexual contact. ${ }^{12}$ Rectal and pharyngeal gonorrhoea has also increased in importance as infection at these sites is more common in homosexual men. Rectal gonorrhoea is recognised as an independent risk factor for HIV infection in homosexual men. ${ }^{34}$ The prevalence of rectal gonorrhoea in men has also been suggested as a rapid method of estimating the amount of unsafe sexual behaviour ${ }^{5}$ and as such can be used to assess the effectiveness of health education and prevention programmes in homosexual men. As HIV infection may also be acquired through oro-genital $\operatorname{sex}^{67}$ the high levels of pharyngeal gonorrhoea, over $50 \%$ recorded in homosexual men in Newcastle ${ }^{1}$ and Edinburgh ${ }^{8}$ are a cause for concern.

Difficulties in the cultural diagnosis of rectal and pharyngeal gonorrhoea include issues of sensitivity, related to low numbers of organ- isms, and specificity, due to the occurrence of non-gonococcal neisseriae such as $N$ meningitidis which are frequently isolated from the throat and occasionally from the rectum. ${ }^{9}$ Non-cultural methods of detecting gonococcal infection should overcome culture-associated problems. The Gen-Probe $\AA$ PACE $₫ 2$ assay uses a chemiluminescent labelled singlestranded DNA probe that is complementary to the ribosomal RNA (rRNA) of the gonococcus: rRNA released from gonococci in the specimen combines with the labelled probe to form a stable DNA:RNA hybrid which is measured objectively in a luminometer. GenProbe has been extensively evaluated using genital specimens and, in general, high sensitivity has been demonstrated with values of $97 \cdot 1 \%,{ }^{10} 99 \cdot 4 \%, 11$ and $100 \%{ }^{12}$ although one recent study reported a sensitivity of $85.9 \%$ for endocervical specimens and $91.5 \%$ for male urethral specimens. ${ }^{13}$ A preliminary evaluation of Gen-Probe gave a sensitivity of $80 \%$ for pharyngeal and $100 \%$ for rectal specimens ${ }^{14}$ 
but it was concluded that further studies were needed.

The objective of this study was to assess the sensitivity and specificity of the Gen-Probe ${ }^{\circledR}$ PACE® 2 assay for the non-cultural detection of rectal and pharyngeal gonorrhoea in homosexual men.

\section{Patients and methods}

Specimens and culture procedures

Duplicate rectal and pharyngeal swabs were collected from 161 homosexual men attending the Genitourinary Medicine Unit, Edinburgh Royal Infirmary during the latter half of 1995 and the first quarter of 1996 . One swab from each site was plated directly onto modified New York City (MNYC) medium ${ }^{15}$ and the plates held at $37^{\circ} \mathrm{C}$ in a carbon dioxide enriched atmosphere for up to four hours before transfer to the laboratory. Cultures were examined after 24 and 48 hours incubation and any suspect colonies tested by the oxidase test: oxidase positive Gram negative diplococci were confirmed as $N$ gonorrhoeae by immunological (Phadebact Monoclonal GC test positive) and biochemical (rapid carbohydrate utilisation) tests ${ }^{16}$ : non-gonococcal neisseriae were speciated on the basis of their carbohydrate utilisation pattern. The other specimen was collected using the Gen-Probe ${ }^{\circledR}$ PACE $® 2$ collection swab and transport tube (Gen-Probe Incorporated, San Diego, California). The order of collecting swabs for culture and Gen-Probe was alternated weekly.

\section{Gen-Probe assays}

The Gen-Probe collection swabs were stored at $-20^{\circ} \mathrm{C}$ and tested according to the manufacturer's instructions. Testing was performed over 20 runs each of which included the three negative reference specimens and the positive control supplied with the kit. The assays were read using the Gen-Probe LEADER Luminometer and the results calculated on the basis of the difference between the response in Relative Light Units (RLU) of the specimen and the mean of the negative reference: a specimen is considered Positive if the difference is $\geqslant \% 300 \mathrm{RLU}$ and negative if the difference is less than 300 RLU.

Repeat testing in the case of discrepant results After comparing the Gen-Probe and culture results all discrepancies were re-tested by GenProbe using the original specimen. Repeatedly reactive Gen-Probe specimens from culture negative patients were also tested by the Gen-

Correlation between culture and Gen-Probe

\begin{tabular}{|c|c|c|c|c|c|c|}
\hline & \multicolumn{2}{|c|}{ Culture positive } & \multicolumn{2}{|c|}{ Culture negative } & \multicolumn{2}{|l|}{ Total } \\
\hline & Rectal & Throat & Rectal & Throat & Rectal & Throat \\
\hline $\begin{array}{l}\text { Gen-Probe positive } \\
\text { Gen-Probe negative } \\
\text { Total }\end{array}$ & $\begin{array}{l}14 \\
11^{\star}\end{array}$ & $\begin{array}{c}9 \\
4 \dagger \\
13\end{array}$ & $\begin{array}{r}3 \ddagger \\
143 \\
146\end{array}$ & $\begin{array}{l}105 \\
138 \\
148\end{array}$ & $\begin{array}{r}17 \\
144 \\
161\end{array}$ & $\begin{array}{r}19 \\
142 \\
161\end{array}$ \\
\hline
\end{tabular}

${ }^{\star}$ Gen-Probe negative on repeat

tOne specimen Gen-Probe positive on repeat

FAll Gen-Probe positive on repeat and confirmed by probe confirmation assay (PCA)

NNine specimens Gen-Probe positive on repeat and confirmed by PCA
Probe competition assay (PCA)..$^{14} 17$ In the PCA assay the sample is tested in duplicate tubes one of which is processed by the standard assay procedure while an excess of probe (identical to the probe used in the standard assay except that it lacks the chemiluminescent label) is added to the second tube. A reduction of $\geqslant \% 70 \%$ in the signal generate in the PCA reaction tube containing unla belled probe compared with the signal gener ated in the standard tube with only labelled probe indicates that the specimen contains gonococci and did not give a reaction due tô? interfering material in the sample. In the case of culture positive Gen-Probe negative speci mens, the cultured gonococci were also testeof by Gen-Probe.

\section{Statistical analysis}

Chi square and two sample $t$ tests were performed with the Minitab PC software package

\section{Results}

Of the 161 patients, $23(14.3 \%)$ gave a posit tive culture at one or both sites (rectum 10 , throat 8 , rectum and throat 5) compared with $28(16.7 \%)$ who gave a positive Gen-Probe result at one or both sites (rectum 9, throat 11 \% rectum and throat 8 ); the number of patient $\mathrm{s}^{\mathrm{N}}$ detected by each method is not statistically significant $\left(\chi^{2}=0.58 ; p>0.3\right)$. The 23 culeg ture positive patients represented infection with seven different serovar/auxotype combi-o nations: IB2/NR (14); IB2/H (4); IB2/P (1) IB3/NR (1); IB7/NR (1); IB8/NR (1); andi IB16/NR (1). Meningococci were isolatedo from $57(35 \cdot 4 \%)$ of the throat cultures, $N$ per flava from $2(1 \cdot 2 \%)$, Moraxella catarrhalis from $2(1 \cdot 2 \%)$, and $N$ lactamica from $1(0 \cdot 6 \%)$. One rectal culture yielded $N$ meningitidis.

The correlation between culture and Gen-? Probe is summarised in the table.

Rectal culture versus Gen-Probe The overal agreement was $97.5 \%(157 / 161)$ with three of the four discrepancies due to negative cul tures. One of these patients had had rectab gonorrhoea three weeks earlier and one had urethral gonorrhoea at the time of sampling while there was no known risk factor in the् case of the third patient. All three positiven Gen-Probe results were repeatedly reactivew and gave $94 \%-97 \%$ inhibition in the PCAO assay confirming that the specimens containedo gonococcal nucleic acid. The Gen-Probe negative (RLU 120) culture positive specimen remained negative (RLU 113) on repeas although a positive probe result was obtained when the cultured isolate, a IB2/NR strain 0 was tested. When Gen-Probe results, without confirmation by PCA, were considered against5 culture the sensitivity, specificity, positive pre응 dictive value (PPV) and negative predictive value (NPV) was $93.3 \%, 97.9 \%, 82.4 \%$ anch $99.3 \%$ respectively. After resolution of discrepant results by PCA and excluding the patient with previous rectal gonorrhoea GenProbe gave a sensitivity, specificity, PPV and NPV of $94 \cdot 1 \%, 100 \%, 100 \%$ and $99 \cdot 3 \%$. The sensitivity of culture was $88.2 \%(15 / 17)$ and 
the NPV was $98 \cdot 6 \%(143 / 145)$ when measured against the confirmed Gen-Probe results. The difference in sensitivity between Gen-Probe and culture is not significant $\left(\chi^{2}\right.$ $1 \cdot 12 ; \mathrm{p}>0 \cdot 2)$.

Pharyngeal culture versus Gen-Probe The overall agreement was $91 \cdot 3 \%(147 / 161)$ with ten of the fourteen discrepancies due to negative cultures. Of the ten patients with positive Gen-Probe results but negative cultures four had rectal gonorrhoea, two were contacts of gonorrhoea, and four had no known risk factors: one of the latter patients was negative on repeat testing. All nine repeat reactive GenProbe specimens (seven with RLU values > 1500) gave $97 \%-99 \%$ inhibition in the PCA assay confirming that the specimens contained gonococcal nucleic acid. One of the four GenProbe negative (RLU 305 against a cut off of 410) culture positive specimens was positive on repeat testing (RLU 403 against a cut-off of 355) while the others remained negative with initial and repeat RLU values below 300. The corresponding isolates from these four patients gave a positive probe result. When Gen-Probe results, without confirmation by PCA, were considered against culture the sensitivity, specificity, PPV and NPV was $69 \cdot 2 \%, 93 \cdot 9 \%$, $50 \%$ and $97.4 \%$ respectively. After resolution of discrepant results by PCA Gen-Probe gave a sensitivity, specificity, PPV and NPV of $86.4 \%, 100 \%, 100 \%$, and $97.9 \%$. The sensitivity of culture was $59 \%(13 / 22)$ and the NPV $93.9 \%(139 / 148)$ when measured against the confirmed Gen-Probe results. The difference in sensitivity between culture and Gen-Probe is significant $\left(\chi^{2}=4 \cdot 13 ; \mathrm{p}<0.05\right)$. The average RLU value for the cut-off was 386 (range 351-450) while the average for the 144 rectal and 142 throat specimens with a negative probe result was 91 and 103 respectively. The average RLU value for a positive result was 20 306 (range 403-110 104): this was, however, significantly higher (two sample $t$ test; $\mathrm{p}=$ 0.019 ) in rectal specimens 31325 (range 1,705-110 104) than in throat specimens 10 447 (range 403-15 633). The corresponding values for culture negative probe positive rectal and throat specimens were 17356 (values 1351,4751 , and 46003 ) and 16,767 (range 414-65 009) respectively. There was no difference between the mean RLU value for the 51 probe negative throat cultures yielding meningococci on culture (101) and the 83 cultures from which meningococci were not isolated (97): the mean RLU values for the two throat cultures yielding $N$ perflava and $M$ catarrhalis were 214 and 87 respectively while the single specimen yielding $N$ lactamica gave a value of 164 .

\section{Discussion}

As the Gen-Probe assay is currently recommended only for urogenital specimens performance data for extra-genital specimens are extremely limited. ${ }^{1014}$ Our results show that Gen-Probe is comparable in sensitivity to rectal culture but is significantly more sensitive than pharyngeal culture and support the earlier sug- gestion that Gen-Probe provides an alternative to culture for rectal and pharyngeal infection. ${ }^{14}$ The sensitivity of culture is not absolute but ranges from $80-95 \%{ }^{18}$ and may be even lower if certain pre-poured selective media are used. ${ }^{19}$ Therefore in order to avoid under-estimating the sensitivity and specificity we re-tested all of the reactive Gen-Probe specimens with a probe competition assay (PCA) although a recent study ${ }^{17}$ found that this was not normally necessary for specimens with RLU values > 1500. We also attempted to use the ligase chain reaction ${ }^{20}$ to corroborate the Gen-Probe results but the specimens were unsuitable for amplification by this method. If the probe results are compared directly with rectal culture without repeat testing or confirmation of the probe result by PCA the sensitivity, specificity, PPV and NPV was $93.3 \%, 97.9 \%$, $82.4 \%$ and $99 \cdot 3 \%$ respectively. However, after confirmation by PCA Gen-Probe gave a sensitivity, specificity, PPV and NPV of $94 \cdot 1 \%$, $100 \%, 100 \%$ and $99.3 \%$ : the sensitivity of culture was reduced to $88 \cdot 2 \%$ and the NPV was $98 \cdot 6 \%$. Comparing probe results directly with pharyngeal culture gave a sensitivity, specificity, PPV and NPV of $69 \cdot 2 \%, 93.9 \%, 50 \%$ and $97.4 \%$ respectively which increased to $86.4 \%, 100 \%, 100 \%$, and $97 \cdot 9 \%$ after confirmation by PCA: the sensitivity of culture was reduced to $59 \%$ and the NPV to $93.9 \%$; the difference in sensitivity between culture and Gen-Probe is significant $(p<0.05)$. The finding that probe positive culture negative pharyngeal results occurred significantly more often $(p<0.001)$ in patients with culture proven rectal gonorrhoea $(26.7 \%)$ than in those without rectal infection (3.4\%) lends epidemiological support to the accuracy of the probe results. False positive probe results due to organisms such as meningococci sharing rRNA sequences to those on which the probe is based are extremely unlikely as the same probe is used in a culture confirmation assay which had absolute specificity when evaluated in an "in-use" assay which included a range of non gonococcal neisseriae. ${ }^{21}$ The finding that GenProbe sensitivity was comparable to rectal culture $(p>0.2)$ but significantly higher than throat culture $(p<0.05)$ is in keeping with the significant difference $(p=0.019)$ in the mean RLU value for rectal (31 325) versus throat specimens (10 447) which indicates a higher antigenic load in the rectal specimens. There were three culture positive Gen-Probe negative pharyngeal specimens compared with one culture positive probe negative rectal specimen which is consistent with sampling differences becoming more important when the number of organisms is low.

It is also possible that Gen-Probe detected non-viable antigen in the pharynx. Serological studies $^{22}$ have suggested that some patients are exposed to pharyngeal gonorrhoea without developing culture-proven pharyngeal infection. $N$ meningitidis has been reported to have a possible protective effect against genital gonococcal infection ${ }^{23}$ so it is possible that a similar protective effect may occur in the pharynx. Nevertheless a positive result would 
appear to indicate exposure to infection and, in the absence of a previously treated infection, could be important in ensuring appropriate partner notification action. There is little information on probe positivity following treatment ${ }^{10}$ and further studies are required to monitor the disappearance of gonococcal nucleic acid after therapy.

Our results demonstrating a prevalence of rectal infection of $\mathbf{1 0 . 6 \%}$ for Gen-Probe versus $8.8 \%$ for culture and a prevalence of pharyngeal infection of $11.8 \%$ for Gen-Probe versus $8.1 \%$ for culture provide a baseline by which other centres could use Gen-Probe to evaluate the efficiency of their rectal and pharyngeal cultures. Whenever possible cultures should be obtained from patients with positive probe test results in order to monitor the antibiotic susceptibility and molecular epidemiology of the gonococcal population.

1 Wardropper A, Pattman RS. An increase in pharyngeal gonorrhoea: cause for concern regarding HIV infection. Genitourin Med 1992;68:343-4

2 Ross JDC, McMillan A, Young H. Increasing incidence of gonorrhoea and syphilis in homosexual men in Edinburgh. Comm Dis Env Health Scotland 1991;25:3-4.

3 Burn S, Horner PI. Rectal gonorrhoea as an independent risk factor for HIV infection in homosexual males. Genitourin Med 1995;71:335-6.

4 Craib KJP, Meddings DR, Strathdee SA, Hogg RS Montaner JSG, Oshaughnessy MV, Schechter MT. Rectal gonorrhoea as an independent risk factor for HIV infection in a cohort of homosexual men. Genitourin Med 1995;71:150-4.

5 Riley VC. Resurgent gonorrhoea in homosexual men. Lancet 1991;19:337:183.

6 Murray AB, Greenhouse PR, Nelson WL, Norman JE, Jeffries DJ, Anderson J. Coincident acquisition of Neifseria gonorrhoeae and HIV from fellatio. Lancet 1991; Neisseria

7 Keet IPM, van Lent NA, Sandfort TGM, Coutinho RA van Griensven JP. Orogenital sex and the transmission of HIV among homosexual men. AIDS 1992;6:223-6.

8 Young H, Moyes A, Ross JDC. Pharyngeal gonorrhoea: an increase in clinical and microbiological significance? In:
Conde-Glez CJ, Morse S, Rice P, Sparling F, Calderon E, eds. Pathobiology and Immunobiology of Neisseriaceae. Mexico: Instituto Nacional de Salud Publica, 1994 140-1.

9 McKenna JG, Fallon RJ, Moyes A, Young H. Anogenital non-gonococcal neisseria: prevalence and clinical significance. Int ₹ STD AIDS 1993;4:8-12.

10 Vlaspolder F, Mutsaers JAEM, Blog F, Notowicz A. Value of a DNA probe assay (Gen-Probe) compared with that of culture for diagnosis of gonococcal infection. $f$ Clin $\mathbb{D}$ Microbiol 1993;31:107-10.

11 Hale YM, Melton ME, Lewis JS, Willis DE. Evaluation of the PACE 2 Neisseria gonorrhoeae assay by three public health laboratories. $f$ Clin Microbiol 1993;31:451-3.

12 Stary A, Kopp W, Zahel B, Nerad S, Teodorowicz L, Horting-Muller I. Comparison of DNA-probe test and culture for the detection of Neisseria gonorrhoeae in genital ${ }^{\circ}$. samples. Sex Transm Dis 1993;20:243-7.

13 Schweble JR, Zajackowski ME. Comparison of DNA probe (Gen-Probe) with culture for the detection ofo probe (Gen-Probe) with culture for the detection ofo Neisseria gonorrhoeae in an urban STD programme.
Genitourin Med 1996;72:108-10.

14 Lewis JS, Fakile O, Foss E, Legarza G, Leskys A, Lowe K, et al. Direct DNA probe assay for Neisseria gonorrhoeae in pharyngeal and rectal specimens. F Clin Microbiol 1993;

15 Young $\mathrm{H}$. Cultural diagnosis of gonorrhoea with modified New York City (MNYC) medium. Br $\mathcal{f}$ Venereal Dis $\mathrm{O}$ 1978;54:36-40.

16 Young H, Moyes A. Utility of monoclonal antibody coag- $\vec{c}$ glutination to identify Neisseria gonorrhoeae. Genitourin Med 1989;65:8-13.

17 Woods GL, Garza DM. Use of Gen-Probe probe competi-= tion assay as a supplement to probes for direct detection of $\omega$ Chlamydia trachomatis and Neisseria gonorrhoeae in urogenital specimens. $\mathcal{F}$ Clin Microbiol 1996;34:177-8.

18 Hook EW, Handsfield HH. Gonococcal infections in the adult. In: Holmes KK, Mardh PA, Sparling PG, et al,음 ds. Sexually Transmitted Diseases. New York: McG al, Hill, 1990:149-65.

19 Young H, Moyes A. An evaluation of pre-poured selective media for the isolation of Neisseria gonorrhoeae. $\mathcal{F}$ Med Microbiol 1996;44:253-60.

20 Smith KR, Ching SF, Lee H, Ohhashi Y, Hu HY, Fisher? $\mathrm{HC}$, et al. Evaluation of ligase chain reaction for use with urine for identification of Neisseria gonorrhoeae in females $\vec{\theta}$ attending a sexually transmitted disease clinic. $\mathcal{F}$ Clin Microbiol 1995;33:455-7.

21 Young $\mathrm{H}$, Moyes A. Comparative evaluation of AccuProbe culture identification test for Neisseria gonorrhoeae ando culture identification test for Neisseria gonorrhoeae and

22 McMillan A, Young H, McNeillage G. Detection of antibodies reactive with Neisseria gonorrhoeae in secretions on extra-genital surfaces. $\mathcal{F}$ Infect 1980;2:53-9.

23 Volk J, Kraus SJ. Asymptomatic meningococcal urethritis. Possible protective value against gonococcal infection by bacteriocin production. BrF Venereal Dis 1978;49:511-2. 